\title{
Aroma profile of a collection of near-isogenic lines of melon (Cucumis melo L.)
}

\author{
Javier M. Obando-Ulloa a, Jorge Ruiz ${ }^{\mathrm{b}, 1}$, Antonio J. Monforte ${ }^{\mathrm{c}, 1}$, J. Pablo Fernández-Trujillo ${ }^{\mathrm{a}, *}$ \\ ${ }^{a}$ Department of Agricultural and Food Engineering, Technical University of Cartagena (UPCT), Campus Paseo Alfonso XIII, 48, \\ ETSIA and Institute of Plant Biotechnology E-30203 Cartagena (Murcia), Spain \\ ${ }^{\mathrm{b}}$ Tecnología de Alimentos, Facultad de Veterinaria, Universidad de Extremadura, Campus Universitario s/n, 10071 Cáceres, Spain \\ ${ }^{\mathrm{C}}$ IRTA, Centre de Recerca en Agrigenòmica CSIC-IRTA-UAB, Ctra de Cabrils, Km 2 E-08348 Cabrils (Barcelona), Spain
}

\section{A R T I C L E I N F O}

\section{Article history:}

Received 8 December 2008

Received in revised form 8 April 2009

Accepted 27 May 2009

Available online $\mathrm{xxxx}$

\section{Keywords:}

Fruit composition

Principal component analysis

Aroma volatiles

Fruit quality

Quantitative trait loci

\begin{abstract}
A B S T R A C T
This paper characterizes the aroma volatile profile found in a collection of near-isogenic lines (NILs) of melon (Cucumis melo L.) obtained by solid-phase microextraction and analyzed by gas chromatography-mass spectrometry. The collection was built with introgressions of an exotic accession (PI 161375) into the parental line 'Piel de sapo' (PS) and was useful for mapping quantitative trait loci (QTLs) associated with melon flesh aroma. The aroma profile was composed of 24 compounds detected in PS or the NILs: three esters, six aldehydes, three alkanes, three aromatic hydrocarbons, two terpenes, two ketones, one alcohol, one sulphur-derived compound, one cyclic branched alkene, one naphthalene and one ether. Hexanal showed the highest relative concentration in the collection, followed by camphor and methanethiolate with no difference between PS and the NILs. These results allowed us to map four QTLs in linkage groups IV, VIII and XI associated with the formation of 3-hydroxy-2,4,4-trimethylpentyl 2-methylpropanoate, octanal and (Z,Z)-3,6-nonadienal.
\end{abstract}

(c) 2009 Elsevier Ltd. All rights reserved.

\section{Introduction}

Melon fruit quality is a multivariate attribute that depends on visual appearance, textural properties, taste and aroma (Beaulieu \& Baldwin, 2002; Obando et al., 2008; Pardo, Alvarruiz, Varón, \& Gómez, 2000; Villanueva, Tenorio, Esteban, \& Mendoza, 2004). Volatile compounds are major determinants of melon fruit quality perceived by consumers, whose acceptance of melon is driven most often by sweetness, sourness, and also by an acceptable aroma bouquet or the presence of volatiles (Beaulieu \& Lea, 2006; Fallik et al., 2001; Kourkoutas, Elmore, \& Mottram, 2006). However, breeding programs have focused on the selection of new vegetable material which produce better colour, size, disease resistance, productivity and other traits, while, only at the end of the process, is any attention paid to aroma (Baldwin, 2002). This is unfortunate since a considerable number of European consumers base their purchases of fruit mainly on the aroma, especially for aromatic melons (Pardo et al., 2000).

Melon aroma is composed of numerous compounds of different degrees of volatility, and is strongly dependent on the cultivar and physiological behaviour of the fruit (Kourkoutas et al., 2006; Obando-Ulloa et al., 2008). Most of the aromatic compounds are esters,

\footnotetext{
* Corresponding author. Tel.: +34 9683254 36; fax: +34 968325433. E-mail addresses: juanp.fdez@upct.es, jf68es@terra.es (J.P. Fernández-Trujillo).

1 Present address: Instituto de Biología Molecular y Celular de Plantas (CSIC-UPV), C/Ingeniero Fausto Elio, s/n, CPI "Ciudad Politécnica de la Innovación"-Edificio 8E, 46022 Valencia, Spain.
}

but aldehydes, alcohols or sulphur-derived compounds have been identified by headspace solid-phase microextraction and analyzed by gas chromatography-mass spectrometry (GC-MS) (Kourkoutas et al., 2006; Obando-Ulloa et al., 2008). The compounds metabolized into aromas are diverse (fatty acids, amino acids, phenols and terpenoids) (Baldwin, 2002), meaning different biochemical pathways for aroma production, such as lipid peroxidation, aldehyde conversion into alcohols, methylerythritol 4-phosphate and mevalonate pathways, fermentation, etc. (Baldwin, 2000, 2002; Beaulieu \& Baldwin, 2002; Ma et al., 2007; Pech, Latché, \& Van der Rest, 2008, chap. 13). These aroma volatile compounds change during fruit ripening, particularly in climacteric fruit, because many of them are ethylene-dependent (Beaulieu, 2006; ObandoUlloa et al., 2008; Pech et al., 2008, chap. 13).

More than 240 compounds (mainly esters) have been identified in climacteric Galia or Cantaloupe melons, but only 42 compounds have been identified in non-climacteric melons such as Rochet, Piel de Sapo, honeydew or Casaba type (Kourkoutas et al., 2006). We established a preliminary profile of 27 probable volatile compounds using 'Piel de sapo' parental (Group Inodorus) and NILs with introgression of the accession PI 161375 (Group Conomon) in the linkage group III (Fernández-Trujillo et al., 2008; Obando-Ulloa et al., 2008). Most of the compounds identified were esters and aldehydes, because most of these NILs were climacteric.

Recent melon aroma research is focused in the elucidation of some of the biosynthetic routes responsible of melon formation by either bioconversion or by tracing of precursors, and to the isolation of the corresponding genes (Pech et al., 2008, chap. 13). 
However, the genetic basis of the aroma formation in melon is still unknown. One strategy to gain knowledge and improve aroma volatiles is the identification of genes or quantitative trait loci (QTLS) associated with certain compounds of the melon aroma profile. This strategy would help to implement quality-oriented breeding programme in order to design fruit with new or improved aroma.

The goal of the present work is to characterize the aroma profile of a collection of NILs that, with PI 161375 introgressions, encompassed many of the twelve linkage groups (LG) of the melon genome. Secondarily, some QTLs associated with aroma volatiles are reported and non-climacteric behaviour verified.

\section{Material and methods}

\subsection{Plant material}

The set of 25 melon near-isogenic lines (NILs) developed by introgressing Korean accession 'Shongwan Charmi' PI 161375 (SC; Group Conomon) genomic regions into 'Piel de sapo' (PS) [C. melo var. inodorus H. Jacq. (Group Inodorus)] genome (Eduardo, Arús, \& Monforte, 2005) used in this study was described by Eduardo et al. (2007). Eighteen NILs contained a single molecular markerdefined introgression from SC into the PS genetic background (Eduardo et al., 2007), and the rest had two or more. The introgressions from SC presented in this set of NILs covered at least $85 \%$ of melon genome. In the present study the NIL SC3 -5 was not used because of its evident climacteric behaviour (Obando-Ulloa et al., 2008). NILs were coded with the prefix "SC" followed by $x-y$ numbers and, in some cases, one or two $z$ letters (SC $x-y z$, for example SC3-5ab): the first $x$ number indicates the linkage group (LG), and the second number $y$ indicates the number of the NIL within the LG. The letters indicates additional genetic facts: " $a$ ", there is more than one introgression; " $b$ " the NIL has an introgression slightly different from the introgression of the NIL with the same number described in Eduardo et al. (2005), although it covers the same genomic region; " $h$ ", the introgression is heterozygous and " $d$ ", part of the introgression is heterozygous (Eduardo et al., 2007). Although the mapping resolution of this population is not very high, it is sufficient for focusing research effort.

\subsection{Experimental design}

Crop management, harvesting practices, and harvesting indices have been previously described (Obando et al., 2008). Nine samples for the parental PS and 86 more for the NILs were analyzed. Only two replicates of NILs SC8-3, SC11-2hab, SC12-4hb and SC11-4d were available for analysis, while three samples of the remaining 21 NILs were analyzed (Table 1 ).

\subsection{Fruit respiration rate}

To confirm climacteric or non-climacteric behaviour, the fruit respiration rate in the NILs of interest was monitored over a period of $14 d$ at $18.6 \pm 1.1{ }^{\circ} \mathrm{C}$ and $68 \pm 9 \%$ relative humidity. Nine NILs and PS were tested according to the static method (Fernández-Trujillo et al., 2008; Obando-Ulloa et al., 2008). The NILs were chosen based on the suspicion of climacteric behaviour in the field.

\subsection{Juice sampling for aroma volatile analysis}

Juice samples were obtained according to Obando-Ulloa et al. (2008) and stored at $-60^{\circ} \mathrm{C}$ until analysis. Juice was squeezed with a Simplex Super metal juicer (Italy) using $20 \mathrm{~mm}$ long and $15 \mathrm{~mm}$ diameter flesh cylinders obtained with an apple cork borer from the middle of one of the longitudinal sections of every fruit. Sam- ples were taken by filtering juice through a powder funnel and four-layer cheesecloth. After $3 \mathrm{~min}, 4 \mathrm{ml}$ of a saturated calcium chloride solution were added to $10 \mathrm{ml}$ of juice and the mixture was homogenized, according to Baldwin, Goodner, Plotto, Pritchett, and Einstein (2004). A 1.33-ml mixture aliquot was poured into 4$\mathrm{ml}$ glass vials for chromatography analysis. These samples were stored in a freezer at $-70{ }^{\circ} \mathrm{C}$ until transportation for eight hours by car to the laboratory in Cáceres (Spain) in a $10 \mathrm{~mm}$-thick polystyrene icebox with eight dry- $\mathrm{CO}_{2}$ tablets of $100 \mathrm{~g}$, and then stored at $-80^{\circ} \mathrm{C}$ until analysis.

\subsection{Volatile analysis: headspace formation, solid-phase micro- extraction (SPME), gas chromatography-mass spectrometry (GC-MS) analysis}

The methodology for gas chromatography-mass spectrometry (GC-MS) analysis of the samples was based on Ruiz, Ventanas, Cava, Andrés, and García (1999) and García-Esteban, Ansorena, Astiasarán, Martín, and Ruiz (2004), taking into account the information supplied by Kourkoutas et al. (2006). The method was described in a previous publication (Obando-Ulloa et al., 2008), but was slightly modified as a result of manual operation. The 4-ml vials were heated at $37^{\circ} \mathrm{C}$ in a stirred shaken water bath for 30 min until equilibrium, and then aroma volatiles were extracted from the headspace for $30 \mathrm{~min}$ with a SPME fibre (StableFlex Divinylbenzene/Carboxen/polydimethyl siloxane $50 / 30 \mu \mathrm{m}$ or DVD/CAR/PDMS) with $1 \mathrm{~cm}$ long standard needle for manual operation (Supelco Ref. 57328-U, Bellefonte, PA, USA), which was previously preconditioned at $280{ }^{\circ} \mathrm{C}$ for $50 \mathrm{~min}$ in the gas chromatography injection port.

After extraction, the fibre was manually introduced in the split/ split-less injector at $280^{\circ} \mathrm{C}$ in order to desorb the aroma volatile compounds during three minutes. The injector was mounted on a HP-6890 gas chromatograph (Agilent Technol., Wilmington, DEL) coupled to a selective single quadrupole mass spectrometer (HP5973 Network, Agilent Technol.). For the split/split-less injector, the liner used had a $0.75 \mathrm{~mm}$ diameter (Supelco, Bellefonte, PA). The volatiles were separated into a $30 \mathrm{~m} \times 0.25 \mathrm{~mm}$ internal diameter with $5 \%$ phenyl-methylsiloxane as stationary phase (HP-5MS, Agilent Technol.). The injection port was in split-less mode and the temperature was isothermal for $10 \mathrm{~min}$ at $40^{\circ} \mathrm{C}$, rising to $200^{\circ} \mathrm{C}$ at $5{ }^{\circ} \mathrm{C} \mathrm{min}-1$, and holding for $5 \mathrm{~min}$. The transfer line to the mass spectrum was maintained at $280^{\circ} \mathrm{C}$.

The mass spectra were obtained by electronic impact at $70 \mathrm{eV}$, a multiplier voltage of $1756 \mathrm{~V}$ and collecting data at a rate of 1 scan $\cdot \mathrm{s}^{-1}$ over the $m / z$ range of $30-500$ AMU. Compounds were tentatively identified by comparing their mass spectra with those included in the National Institute for Standards and Technology (NIST98, search version 2.0) data bank and by comparison of Kovats indices with those reported in the literature (Kondjoyan \& Berdagué, 1996). The retention times from a series of straightchain alkanes (C8-C20) supplied by Fluka were used under identical conditions to calculate the Kovats indices for all the identified volatile compounds (Kondjoyan \& Berdagué, 1996; for "DB-5 like" phases). C6 and C7 were calculated from compounds present in our chromatograms. In addition, the Chemical Abstract Service (CAS) numbers of the volatiles reported in the NIST98 database also found in the website http://webbook.nist.gov/chemistry/nameser.html (Table 2), were used to obtain their corresponding IUPAC names by checking the website http://www.chemindustry.com/ apps/chemicals (Obando-Ulloa et al., 2008). The results of the volatile analyzes were expressed as percentage of total chromatographic area of the 28 volatile compounds of the profile reported in Table 2 (Muriel, Antequera, Petrón, Andrés, \& Ruiz, 2004). These compounds were recovered and positively identified within the run time (Beaulieu, 2005, 2006; Muriel et al., 2004). 
Table 1

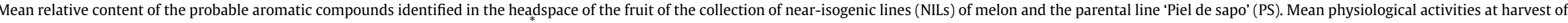
the fruit analyzed are also reported. NIL means within rows highlighted with showed statistical differences from PS data, according to a Dunnett's test at $P=0.05$.

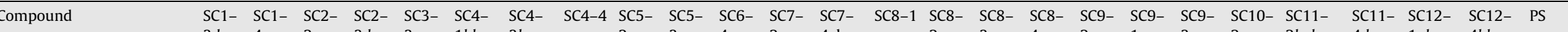

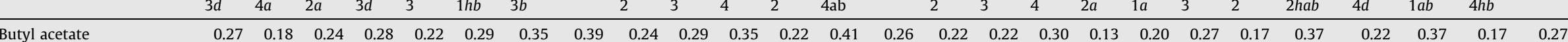

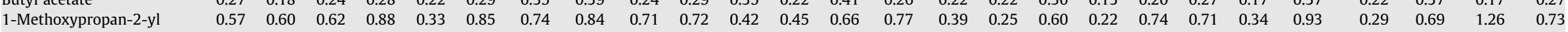

acetate

3-Hydroxy-2,4,4-

trimethylpentyl

2-methylpropanoate

Pentanal

Hexanal

Heptanal

Benzaldehyde

Octanal

Decanal

2-Methylpentane

3-Methylpentane

Methylcyclopentane

Ethylbenzene

1,4-Dimethylbenzene

1,2-Dimethylbenzene

4,7,7-

Trimethylbicyclo[3.1.1]

-3-heptene

Terpene not identified (41

6995108119137152

6-Methyl-5-heptene-2-on

5,5,6-Trimethyl-, endo-

bicyclo[2.2.1]

-2-heptanone

Methanethiol

Ethanol

Ethyl ether

$\begin{array}{llll}0.29 & 0.69 & 1.26 & 0.73\end{array}$

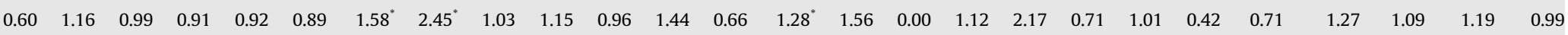

$\begin{array}{llllllllllllllllllllll}2.69 & 3.58 & 2.14 & 2.26 & 2.14 & 2.79 & 2.23 & 0.86 & 1.88 & 2.09 & 3.32 & 1.10 & 1.52 & 2.71 & 1.35 & 2.43 & 0.94 & 0.50 & 3.43 & 2.96 & 2.67 & 4.96\end{array}$

$\begin{array}{rrrrrrrrrrrrrrrrrrrrrrrrrr}29.96 & 39.87 & 25.18 & 25.97 & 27.44 & 33.63 & 31.01 & 8.40 & 22.21 & 22.30 & 30.94 & 15.37 & 22.01 & 30.39 & 16.06 & 28.76 & 12.27 & 10.83 & 42.05 & 33.41 & 32.56 & 48.95 & 28.24 & 4.98 & 21.48 & 29.53\end{array}$

$\begin{array}{llllllllllllllllllllllllll}1.88 & 3.07 & 3.05 & 2.21 & 2.12 & 2.19 & 3.09 & 1.32 & 1.96 & 1.78 & 3.51 & 1.41 & 2.00 & 3.54 & 1.61 & 2.00 & 1.31 & 0.59 & 2.16 & 3.00 & 1.71 & 1.94 & 2.18 & 0.66 & 2.11 & 2.74\end{array}$

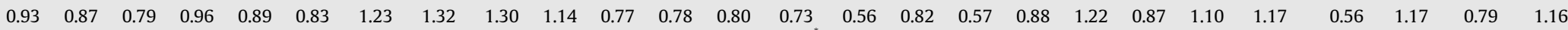

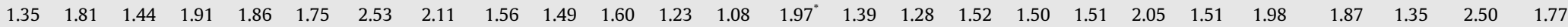

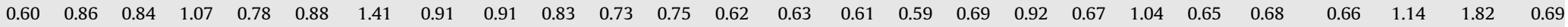

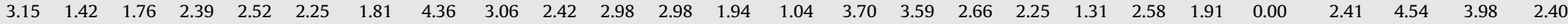
$\begin{array}{llllllllllllllllllllllllll}1.14 & 1.26 & 1.51 & 2.16 & 1.93 & 2.09 & 1.96 & 3.96 & 2.78 & 2.46 & 2.99 & 3.13 & 1.55 & 1.18 & 3.60 & 3.53 & 2.64 & 3.14 & 1.42 & 1.74 & 1.78 & 0.86 & 2.41 & 2.57 & 4.06 & 2.18\end{array}$

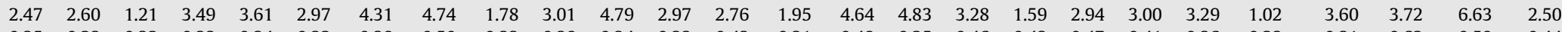
$\begin{array}{lllllllllllllllllllllllllll}0.35 & 0.23 & 0.32 & 0.33 & 0.34 & 0.32 & 0.38 & 0.50 & 0.33 & 0.38 & 0.34 & 0.33 & 0.43 & 0.31 & 0.48 & 0.25 & 0.46 & 0.43 & 0.47 & 0.41 & 0.36 & 0.23 & 0.31 & 0.62 & 0.59 & 0.44\end{array}$

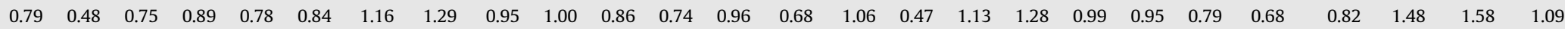
$\begin{array}{llllllllllllllllllllllllll}0.48 & 0.38 & 0.43 & 0.46 & 0.42 & 0.46 & 0.62 & 0.73 & 0.62 & 0.55 & 0.43 & 0.45 & 0.48 & 0.50 & 0.53 & 0.30 & 0.58 & 0.61 & 0.56 & 0.56 & 0.43 & 0.53 & 0.48 & 0.73 & 0.69 & 0.54\end{array}$ $\begin{array}{llllllllllllllllllllllllll}0.29 & 0.12 & 0.18 & 0.26 & 0.17 & 0.21 & 0.28 & 0.39 & 0.25 & 0.21 & 0.26 & 0.24 & 0.29 & 0.14 & 0.32 & 0.21 & 0.22 & 0.29 & 0.15 & 0.24 & 0.12 & 0.21 & 0.17 & 0.29 & 0.28 & 0.25\end{array}$

1-Methyl-4-prop-1-en-

(D-limone)

1,7,7-Trimethylnorbornan-

2-one (Camphor)

Respiration rate

(nmol kg-1 $\left.\mathrm{s}^{-1}\right)$

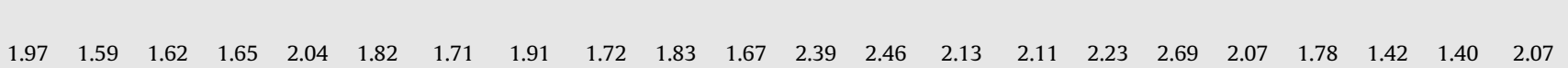

$\begin{array}{llllllllllllllllllllll}1.97 & 1.59 & 1.62 & 1.65 & 2.04 & 1.82 & 1.71 & 1.91 & 1.72 & 1.83 & 1.67 & 2.39 & 2.46 & 2.13 & 2.11 & 2.23 & 2.69 & 2.07 & 1.78 & 1.42 & 1.40 & 2.07\end{array}$

$\begin{array}{llllllllllllllllllllll}0.53 & 0.93 & 0.72 & 1.07 & 0.73 & 0.94 & 1.60 & 1.20 & 0.95 & 1.09 & 0.68 & 0.88 & 0.93 & 0.79 & 0.75 & 0.69 & 1.21 & 0.87 & 0.60 & 0.93 & 0.53 & 0.87\end{array}$

$2.11+\frac{10}{2.88} \quad 0.67 \quad 2.19$

$\begin{array}{llll}2.11 & 2.88 & 0.67 & 2.19\end{array}$

$\begin{array}{llllllllllllllllllllllllll}0.31 & 0.46 & 0.43 & 0.54 & 0.38 & 0.24 & 0.87 & 0.49 & 0.48 & 0.45 & 0.30 & 0.30 & 0.30 & 0.49 & 0.42 & 0.43 & 0.21 & 0.87 & 0.60 & 0.93 & 0.53 & 0.87 & 0.63 & 0.99 & 1.62 & 0.87\end{array}$

$\begin{array}{rrrrrrrrrrrrrrrrrrrrrrrrrrr}12.47 & 8.77 & 14.01 & 12.30 & 8.82 & 12.49 & 6.64 & 11.66 & 13.34 & 11.73 & 7.75 & 14.06 & 7.61 & 10.51 & 13.90 & 10.91 & 15.74 & 10.43 & 7.35 & 10.96 & 12.43 & 9.41 & 8.26 & 13.73 & 17.47 & 10.59\end{array}$ $\begin{array}{rrrrrrrrrrrrrrrrrrrrrrrrrrrr}5.27 & 5.77 & 5.40 & 8.19 & 10.13 & 8.12 & 11.35 & 13.77 & 10.60 & 8.55 & 7.76 & 9.14 & 12.39 & 5.68 & 9.02 & 7.93 & 6.67 & 6.99 & 4.35 & 8.43 & 6.68 & 0.22 & 10.45 & 5.60 & 12.50 & 6.37\end{array}$ $\begin{array}{llllllllllllllllllllllllllll}1.60 & 4.45 & 5.50 & 4.12 & 7.68 & 3.15 & 3.12 & 5.66 & 5.55 & 4.50 & 8.43 & 5.76 & 3.25 & 5.61 & 7.37 & 9.24 & 4.42 & 6.41 & 5.37 & 4.28 & 6.25 & 1.14 & 5.00 & 5.62 & 6.69 & 4.56 \\ 0.34 & 0.52 & 0.50 & 0.44 & 0.60 & 0.58 & 0.64 & 0.62 & 0.39 & 0.47 & 0.38 & 0.56 & 0.40 & 0.36 & 0.54 & 0.38 & 0.60 & 0.70 & 0.45 & 0.53 & 0.49 & 0.31 & 0.52 & 0.58 & 0.67 & 0.42\end{array}$ Ethylene production

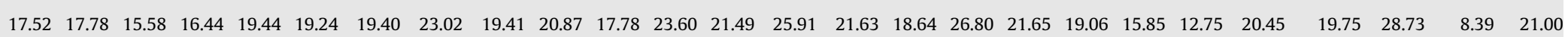
$\begin{array}{lllllllllllllllllllllllllll}71.61 & 63.41 & 64.13 & 79.99 & 66.21 & 54.40 & 85.77 & 71.14 & 66.25 & 63.99 & 61.14 & 77.51 & 77.44 & 66.06 & 76.26 & 71.98 & 68.99 & 72.85 & 72.76 & 56.82 & 48.53 & 56.63 & 59.43 & 63.08 & 76.83 & 65.19\end{array}$ $\begin{array}{llllllllllllllllllllllllllllll}19.20 & 18.10 & 12.00 & 14.70 & 17.21 & 19.71 & 29.02 & 12.93 & 19.59 & 12.76 & 12.98 & 17.60 & 18.71 & 13.42 & 14.90 & 10.72 & 17.29 & 17.49 & 18.70 & 20.28 & 18.31 & 10.29 & 14.81 & 12.83 & 16.40 & 16.20\end{array}$ $\left(\right.$ (nnolk $\left.\mathrm{kg}^{-1}\right)$ 
Table 2

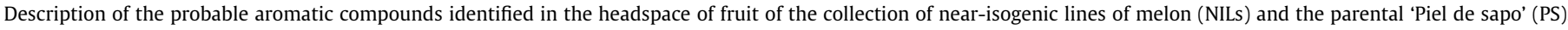

\begin{tabular}{|c|c|c|c|c|}
\hline Compound's name & No. CAS & Mass-to-charge ratio $(\mathrm{m} / \mathrm{z})$ & KI & ID \\
\hline \multicolumn{5}{|l|}{ Esters } \\
\hline Butyl acetate & $123-86-4$ & $\begin{array}{l}43 \text { (999), } 56 \text { (442), } 41 \text { (147), } 61 \text { (144), } 73 \text { (142), } 55 \text { (74), } 29 \text { (66), } 27 \text { (50), } 57 \text { (41), } 15 \\
(36)\end{array}$ & 815 & $\mathrm{a}$ \\
\hline 1-Methoxypropan-2-yl acetate & $108-65-6$ & $\begin{array}{l}43 \text { (999), } 45 \text { (497), } 15 \text { (239), } 72 \text { (203), } 29 \text { (91), } 58 \text { (120), } 41 \text { (90), } 87 \text { (87), } 27 \text { (65), } 39 \\
\text { (58) }\end{array}$ & 870 & $a, b$ \\
\hline $\begin{array}{l}\text { 3-Hydroxy-2,4,4-trimethylpentyl } \\
\text { 2-methylpropanoate }\end{array}$ & $74367-34-3$ & $\begin{array}{l}71 \text { (999), } 56 \text { (756), } 43 \text { (609), } 89 \text { (455), } 41 \text { (235), } 55 \text { (203), } 73 \text { (162), } 57 \text { (162), } 72 \text { (89), } \\
85 \text { (81) }\end{array}$ & 1389 & $\mathrm{a}, \mathrm{b}$ \\
\hline \multicolumn{5}{|l|}{ Aldehydes } \\
\hline Pentanal & $110-62-3$ & $\begin{array}{l}44 \text { (999), } 29 \text { (690), } 41 \text { (522), } 27 \text { (461), } 43 \text { (311), } 58 \text { (282), } 39 \text { (268), } 57 \text { (257), } 28 \text { (192), } \\
42 \text { (151) }\end{array}$ & 696 & $\mathrm{a}, \mathrm{b}$ \\
\hline Hexanal & $66-25-1$ & $\begin{array}{l}44 \text { (999), } 56 \text { (816), } 41 \text { (655), } 43 \text { (531), } 57 \text { (441), } 29 \text { (382), } 27 \text { (330), } 72 \text { (197), } 45 \text { (196), } \\
55 \text { (147) }\end{array}$ & 799 & $\mathrm{a}, \mathrm{b}$ \\
\hline Heptanal & $111-71-7$ & $\begin{array}{l}44 \text { (999), } 43 \text { (861), } 41 \text { (844), } 70 \text { (662), } 27 \text { (611), } 42 \text { (555), } 55 \text { (512), } 57 \text { (423), } 39 \text { (287), } \\
45 \text { (204) }\end{array}$ & 900 & a,b \\
\hline Benzaldehyde & $100-52-7$ & $\begin{array}{l}77 \text { (999), } 106 \text { (944), } 105 \text { (944), } 51 \text { (384), } 50 \text { (185), } 78 \text { (139), } 52 \text { (93), } 107 \text { (72), } 74 \text { (62), } \\
39 \text { (62) }\end{array}$ & 965 & $\mathrm{a}, \mathrm{b}$ \\
\hline Octanal & $124-13-0$ & $\begin{array}{l}41 \text { (999), } 43 \text { (883), (57) 809, } 56 \text { (785), } 55 \text { (690), } 44 \text { (684), } 84 \text { (564), } 29 \text { (478), } 69 \text { (418), } \\
42 \text { (418) }\end{array}$ & 1000 & $\mathrm{a}, \mathrm{c}$ \\
\hline Decanal & $112-31-2$ & $\begin{array}{l}43 \text { (999), } 41 \text { (985), } 57 \text { (785), } 55 \text { (549), } 44 \text { (542), } 29 \text { (451), } 70 \text { (380), } 56 \text { (351), } 42 \text { (342), } \\
71 \text { (333) }\end{array}$ & 1209 & $\mathrm{a}, \mathrm{c}$ \\
\hline \multicolumn{5}{|l|}{ Branched alkanes } \\
\hline 2-Methylpentane & $107-83-5$ & $\begin{array}{l}43 \text { (999), } 42 \text { (528), } 41 \text { (354), } 27 \text { (305), } 71 \text { (285), } 39 \text { (200), } 29 \text { (179), } 57 \text { (106), } 15 \text { (95), } \\
70 \text { (67) }\end{array}$ & 558 & $\mathrm{a}$ \\
\hline 3-Methylpentane & $96-14-0$ & $\begin{array}{l}57 \text { (999), } 56 \text { (756), } 41 \text { (675), } 29 \text { (604), } 27 \text { (398), } 43 \text { (292), } 39 \text { (218), } 15 \text { (89), } 55 \text { (88), } 28 \\
(82)\end{array}$ & 576 & $\mathrm{a}, \mathrm{b}$ \\
\hline Methylcyclopentane & $96-37-7$ & $\begin{array}{l}56 \text { (999), } 41 \text { (547), } 69 \text { (438), } 39 \text { (263), } 55 \text { (261), } 42 \text { (201), } 28 \text { (160), } 27 \text { (159), } 84 \text { (106), } \\
43 \text { (79) }\end{array}$ & 622 & $\mathrm{a}, \mathrm{c}$ \\
\hline \multicolumn{5}{|l|}{ Aromatic hydrocarbon } \\
\hline Ethylbenzene & $100-41-4$ & $\begin{array}{l}91 \text { (999), } 106 \text { (282), } 51 \text { (114), } 65 \text { (113), } 77 \text { (99), } 78 \text { (87), } 92 \text { (73), } 39 \text { (70), } 50 \text { (62), } 105 \\
\text { (61) }\end{array}$ & 866 & $\mathrm{a}, \mathrm{c}$ \\
\hline 1,4-Dimethylbenzene & $106-42-3$ & $\begin{array}{l}91 \text { (999), } 106 \text { (658), } 105 \text { (288), } 77 \text { (118), } 51 \text { (85), } 39 \text { (79), } 92 \text { (74), } 79 \text { (70), } 103 \text { (58), } 78 \\
\text { (57) }\end{array}$ & 873 & $\mathrm{a}, \mathrm{c}$ \\
\hline 1,2-Dimethylbenzene & $95-47-6$ & $\begin{array}{l}91 \text { (999), } 106 \text { (430), } 105 \text { (177), } 51 \text { (135), } 39 \text { (135), } 77 \text { (129), } 65 \text { (87), } 79 \text { (82), } 78 \text { (72), } \\
27 \text { (68) }\end{array}$ & 896 & $\mathrm{a}, \mathrm{c}$ \\
\hline \multicolumn{5}{|l|}{ Terpenes } \\
\hline $\begin{array}{l}\text { 4,7,7-Trimethylbicyclo[3.1.1] -3- } \\
\text { heptene }\end{array}$ & $\begin{array}{l}\text { Not previously } \\
\text { identified }\end{array}$ & & 940 & $\mathrm{a}, \mathrm{b}$ \\
\hline $\begin{array}{l}\text { Terpene not identified (41 } 6995 \\
\qquad 108119137152)\end{array}$ & $\begin{array}{l}\text { Not previously } \\
\text { identified }\end{array}$ & & 1118 & $\mathrm{a}$ \\
\hline \multicolumn{5}{|l|}{ Ketones } \\
\hline 6-Methyl-5-heptene-2-one & $110-93-0$ & $\begin{array}{l}43 \text { (999), } 41 \text { (460), } 69 \text { (341), } 55 \text { (330), } 108 \text { (276), } 58 \text { (171), } 111 \text { (165), } 68 \text { (153), } 39 \\
\text { (130), } 71 \text { (129) }\end{array}$ & 985 & $\mathrm{a}, \mathrm{c}$ \\
\hline $\begin{array}{l}\text { 5,5,6-Trimethyl-, endo- } \\
\text { bicyclo[2.2.1] -2-heptanone }\end{array}$ & $3767-44-0$ & $\begin{array}{l}108 \text { (999), } 95 \text { (793), } 41 \text { (503), } 55 \text { (448), } 69 \text { (348), } 39 \text { (257), } 67 \text { (254), } 109 \text { (254), } 81 \\
(242), 110(242)\end{array}$ & 1215 & $\mathrm{a}$ \\
\hline \multicolumn{5}{|l|}{ Other compounds } \\
\hline Methanethiol & 74-93-1 & $\begin{array}{l}47 \text { (999), } 48 \text { (758), } 45 \text { (614), } 46 \text { (147), } 44 \text { (126), } 33 \text { (121), } 32 \text { (116), } 14 \text { (67), } 49 \text { (56), } 13 \\
(43)\end{array}$ & ND & $\mathrm{a}, \mathrm{c}$ \\
\hline Ethanol & $64-17-5$ & $\begin{array}{l}31 \text { (999), } 45 \text { (514), } 29 \text { (298), } 27 \text { (224), } 46 \text { (216), } 43 \text { (114), } 26 \text { (98), } 30 \text { (81), } 15 \text { (66), } 42 \\
(47)\end{array}$ & ND & $\mathrm{a}$ \\
\hline Ethyl ether & $60-29-7$ & $\begin{array}{l}31 \text { (999), } 29 \text { (627), } 59 \text { (396), } 27 \text { (346), } 45 \text { (326), } 74 \text { (225), } 15 \text { (174), } 43 \text { (90), } 26(88), 28 \\
(85)\end{array}$ & 504 & $\mathrm{a}, \mathrm{b}$ \\
\hline $\begin{array}{l}\text { 1-Methyl-4-prop-1-en-2-yl- } \\
\text { cyclohexene (D-limone) }\end{array}$ & $5989-27-5$ & $\begin{array}{l}68 \text { (999), } 93 \text { (590), } 67 \text { (446), } 79 \text { (227), } 94 \text { (225), } 136 \text { (225), } 121 \text { (194), } 41 \text { (192), } 92 \\
(187), 107 \text { (170) }\end{array}$ & 1034 & $\mathrm{a}, \mathrm{b}$ \\
\hline $\begin{array}{l}\text { 1,7,7-Trimethylnorbornan-2-one } \\
\quad \text { (Camphor) }\end{array}$ & $76-22-2$ & $\begin{array}{l}95 \text { (999), } 41 \text { (794), } 81 \text { (739), } 108 \text { (388), } 69 \text { (386), } 55 \text { (376), } 27 \text { (348), } 39 \text { (335), } 83 \text { (331), } \\
109 \text { (282) }\end{array}$ & 1163 & $\mathrm{a}$ \\
\hline
\end{tabular}

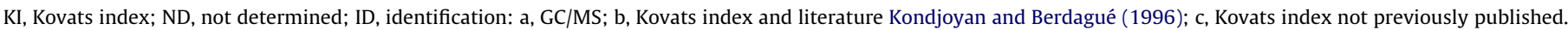

\subsection{Statistical analysis}

Data were subjected to a one-way ANOVA using general linear model procedures, with pedigree as factor in JMP ${ }^{\circledR}$ v5.1.2 for Windows (SAS Institute Inc., NC). If significant differences were found by ANOVA using pedigree, the differences between NILs and PS were evaluated by Dunnett's test at $P=0.05$, using JMP, according to Obando et al. (2008).

Aroma data were also subjected to principal components analysis (PCA), using JMP. This analysis was used to identify the volatiles that explain the most part of the volatile differences among NILs and to group the NILs according their aroma profile. The NIL PC values of all the compounds were analyzed by ANOVA with pedigree as factor and Dunnett's test in JMP. The PCA graph was split into two graphs (score and loading plots, respectively) in order to clarify the analysis due to the high number of samples and volatiles considered in this study. Otherwise, the PCA figure with the score plot overlapping the loading plot would be a cloud of data with no sense. The score plot was performed with the mean component value (prin) obtained for each PC, while the loading plot was reached with the eigenvectors greater than 0.20 for the aroma volatile compounds that better define the orientation of each PC axis. 


\section{Results}

\subsection{Respiration rate}

The PS showed a non-climacteric pattern, while four NILs showed a similar non-climacteric behaviour (Fig. 1). Compared with the respiration rate levels of PS, SC8-4 and SC9-2a showed slightly higher levels, while SC2-3d showed slightly lower levels. The NIL SC3-3 was classified as having a slightly climacteric behaviour (Fig. 1). Other NILs checked at harvest (Table 1) or in other experiments, such as SC10-2, also showed the levels of non-climacteric PS or a typical non-climacteric pattern (data not shown).

\subsection{Definition of the aroma profile}

About 206 aroma volatile compounds were tentatively identified in the headspace of the NILs analyzed, although only 48 compounds were preliminarily identified in most of the NILs and in PS. The definition of the aroma profile was based on the number of samples in which the compound was detected. So, in cases in which there were two NIL samples and the compound was detected in only one of them, the compound was not considered for the profile. On the other hand, if there were three samples and the compound was identified in two of the three or in all three samples, it was considered for the profile. That is why only 24 compounds defined the probable aroma profile in the whole collection and PS (Table 1). The profile was composed of six aldehydes, three esters, three branched alkanes, three aromatic branched hydrocarbons, two terpenes, two ketones and five compounds from other groups (Tables 1 and 2 ).

It was also found that $(Z, Z)-3,6$ nonadienal was only detected in NIL SC8-1, with a relative content levels of around $0.51 \%$.

\subsection{Principal component analysis of the aroma volatile data}

PCA transformed a number of possibly correlated variables of single aroma volatiles into a smaller number of uncorrelated variables called principal components. Principal components were obtained as linear combinations of the original variables, so the first principal component has maximum possible variance, the second principal component is orthogonal to the first principal component and has the maximum possible variance and so on. PC components explain better the melon aroma than single compounds individually. The first three PCs contributed to $57 \%$ of the total variance
(30\%, 15\% and 12\%, respectively). Fig. 2A depicts PC1 versus PC2. The parental line PS was located close to the centre of the graph. The NIL SC12-1ab showed the higher PC1 levels, significantly higher than PS, according to the Dunnett's test (Table 1; data not shown). NILs SC4-3b, SC11-2hab showed higher PC2 values than PS, while SC7-2 showed lower PC2 values according to Dunnett's tests (Fig. 2A; data not shown).

PC1, representing $30 \%$ of the total variance, was mostly affected by the relative concentrations of 2-methylpentane, 3-methylpentane, ethylbenzene, 1,4-dimethylbenzene, 1,2-dimethylbenzene and 5,5,6-trimethyl-, endo-bicyclo[2.2.1]-2-heptanone in the positive direction, while pentanal, hexanal, and heptanal defined the negative orientation of the PC1 axis (Fig. 2B). PC2, representing $15 \%$ of the total variance, was affected by relative concentrations of methanethiol, ethanol, ethyl ether, methylcyclopentane, which defined its negative orientation, while its positive orientation was defined by 1 -methoxypropan-2-yl acetate, 6 -methyl-5-heptene-2-one, octanal and decanal. The third component PC 3 , representing $12 \%$ of the total variance, was mainly affected in its negative orientation by the relative concentration of butyl acetate, a non-identified terpene and 1,7,7-trimethylnorbornan-2-one (camphor) (data not shown).

\subsection{Univariate analysis of the compounds identified in the aroma profile}

Three compounds of the profile [hexanal, 1,7,7-trimethylnorbornan-2-one (camphor), and methanethiol] showed noticeably higher levels than the rest of the compounds but with no significant pedigree effect.

However, compounds with lower relative content were more affected by the pedigree factor. Some of them, such as the 3-hydroxy-2,4,4-trimethylpentyl 2-methylpropanoate, showed a higher relative content compared with PS (29-148\% in the NILs SC4-3b, SC4-4 and SC11-4d). On the other hand, octanal showed a $11.3 \%$ lower level in the NIL SC8-1 than PS (Table 1).

\subsection{QTL mapping}

Only three QTLs were associated with the aroma profile of these NILs. Two of them increased the ester 3-hydroxy-2,4,4-trimethylpentyl 2-methylpropanoate (2 QTLs in LG IV and XI), while other in LG VIII reduced the relative content of octanal. Additionally, another QTL in LG VIII was responsible for the cucumber-like aroma [(Z,Z)-3,6 nonadienal], characteristic of NIL SC8-1, as mentioned above.
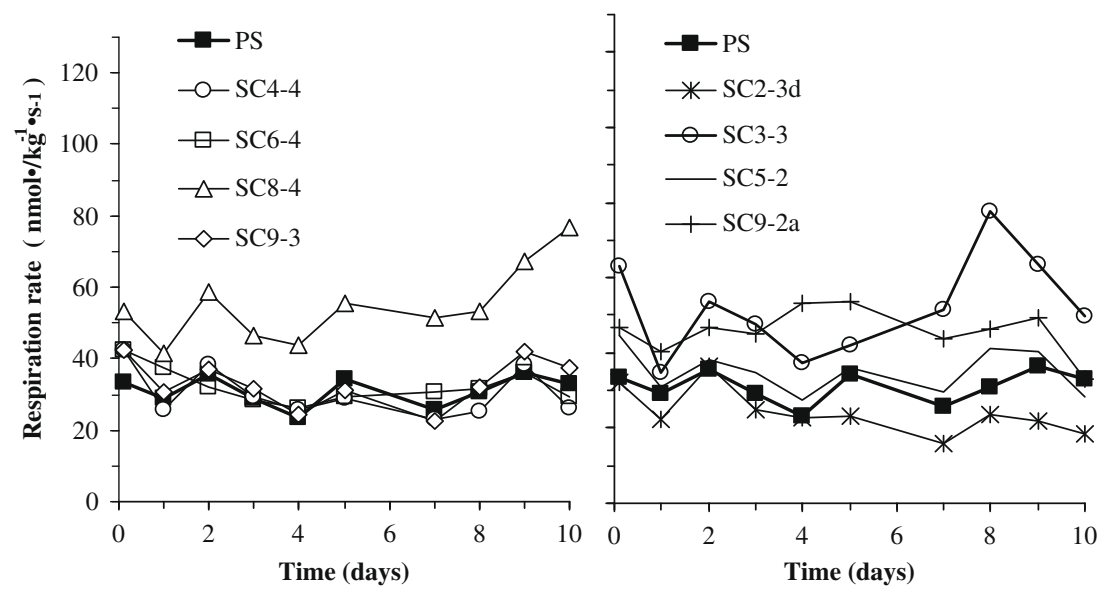

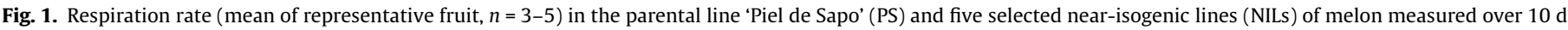
at $18.6^{\circ} \mathrm{C}$. 

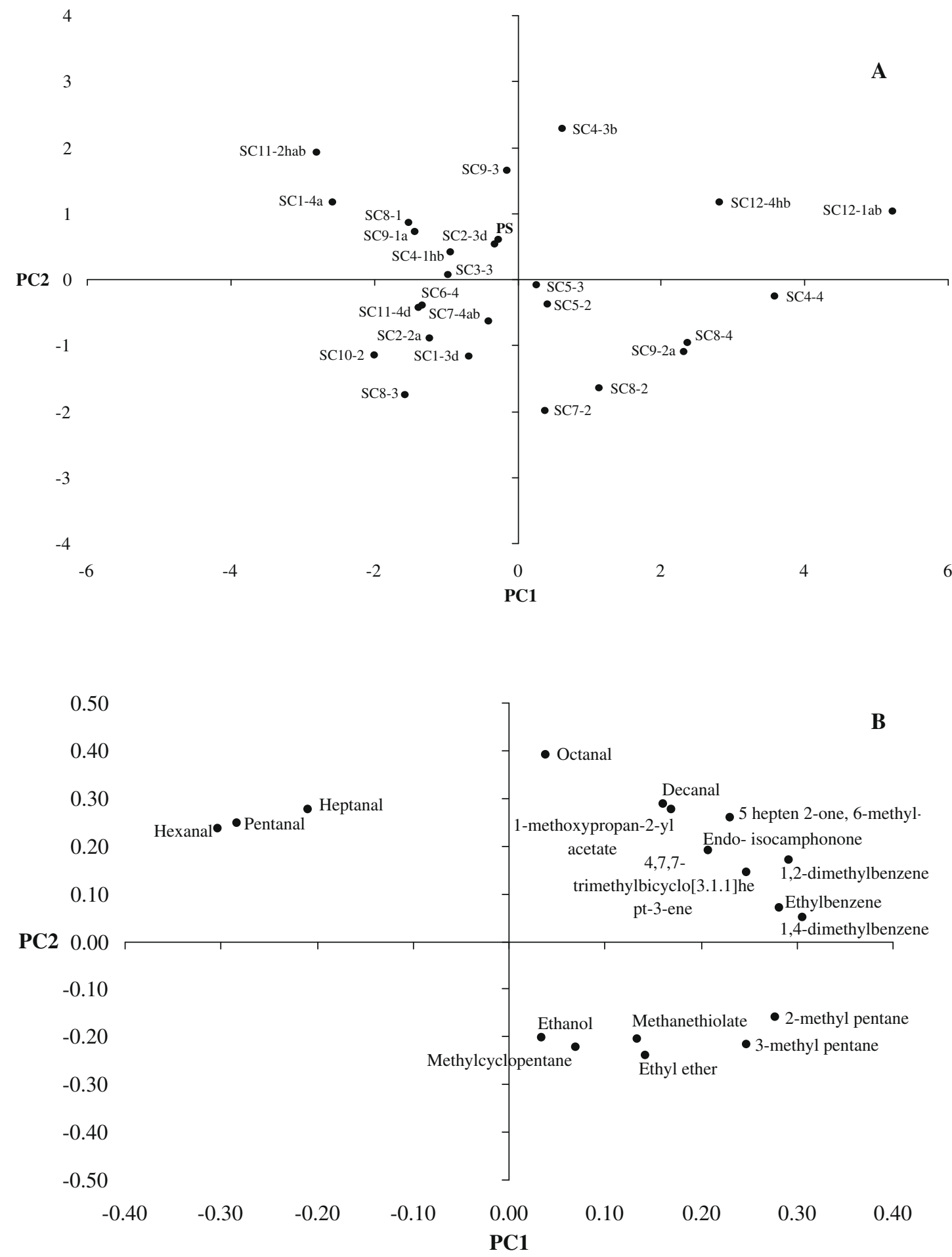

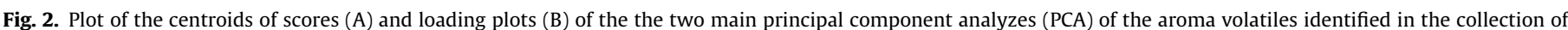

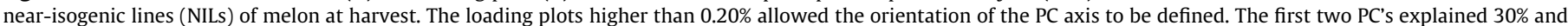
$15 \%$ of the total variance, respectively. The biplot was divided in two figures in order to provide a clearer graph for a better understanding of the results.

\section{Discussion}

Overall, PI 161375 did not really affect the physiological behaviour of the NILs studied, which showed a non-climacteric behaviour, except NIL SC8-4 with its respiration rate that seemed to be higher and showed a trend to increase (Fig. 1), and the NIL SC3-5 previously studied (Moreno et al., 2008; Obando-Ulloa et al., 2008; data not shown). The aroma profile of non-climacteric Inodorous melons has received little attention in the literature
(Kourkoutas et al., 2006), but these melons have great interest as intact, fresh-cut or processed fruit due to their longer shelf-life than the climacteric ones (Beaulieu \& Baldwin, 2002; FernándezTrujillo et al., 2008; Oms-Oliu, Odriozola-Serrano, Soliva-Fortuny, \& Martin-Belloso, 2008).

Usually, ethylene burst aroma biosynthesis is easy to detect by the human nose, while in non-climacteric fruit the external aroma is scarcely detected except when the fruit become senescent or rotten (Obando-Ulloa et al., 2009). The non-climacteric PS type, how- 
ever, shows many aroma compounds, most of them aldehydes (Table 1). Even though some aldehydes are not easily detectable by the human nose, others have a low human detection threshold (Baldwin, 2002; Beaulieu \& Baldwin, 2002; Canoles, Beaudry, Li, \& Howe, 2006), and are important for the flavour of tomato, cucumber, peppers and other vegetables (Baldwin, 2002; Canoles et al., 2006). Aldehydes with low molecular weight are associated with immature stages in climacteric Cantaloupe melons (Beaulieu \& Grimm, 2001). However, most aldehydes are present at high levels or increase markedly during early growth stages and then decrease with increasing harvest maturity (Beaulieu \& Grimm, 2001). In this collection, aldehydes are present in between $15-40 \%$ relative levels of the total aroma of the profile, and hexanal shows the highest relative content with respect to other aldehydes, in agreement with previous results working with PS and other non-climacteric NILs with introgression in LG III (Obando-Ulloa et al., 2008). Hexanal is considered to contribute to green notes and, secondarily, to melon aroma, and benzaldehyde has been defined as a pleasant aroma for Cantaloupe type melons (Beaulieu, 2006; Beaulieu \& Grimm, 2001), and high concentrations are usually associated with a high degree of melon ripening (Beaulieu \& Grimm, 2001). Also, it is not unlikely that the unsaturated aldehyde fraction and, particularly, hexanal, may be due to lipid peroxidation during sampling and freezing (Ma et al., 2007). It has also been reported that hexanal increases with increasing harvest maturity, which could lead to development of off-flavours in stored melon and fresh-cut products (Beaulieu \& Grimm, 2001) and in tomato (Canoles et al., 2006). However, hexanal slightly changes during PS senescence compared with other NILs (Obando-Ulloa et al., 2009).

The rest of the main compounds of the profile have previously been reported as aroma components in other foods. For example, methanethiolate has been associated with food heating and/or cooking, and is also an essential compound for the flavour of cured ham (Ruiz et al., 1999). However, the high concentrations in some NILs and reference cultivars seem to contribute to the musky notes of the aroma (Obando-Ulloa et al., 2008).

On the other hand, 1,7,7-trimethylnorbornan-2-one (camphor) have also been reported in other melon cultivars (Lamikanra \& Richard, 2002), and in NILs with introgression of the melon LG III (Obando-Ulloa et al., 2008). The three QTLs mapped for three different compounds and LG's can be used as a good model for melons to study the relationship between aroma biosynthesis and aroma precursors (Baldwin, 2002). Octanal is an aldehyde previously reported as a major component $(12.7 \% \mathrm{v} / \mathrm{v})$ in orange flavourings (Muñoz-Aguirre, Yoshino, Nakamoto, \& Morizumi, 2007), but also reported in the flesh of Queen Ann's pocket melons (Aubert \& Pitrat, 2006), fresh-cut Cantaloupe melons (Beaulieu \& Lancaster, 2007), in and in seedless watermelon juice (Beaulieu \& Lea, 2006).

The compound 3-hydroxy-2,4,4-trimethylpentyl 2-methylpropanoate is a complex ester not previously reported in PS or the NILs studied with introgression in LG III (Obando-Ulloa et al., 2008). This compound has been found in green tea (Hattori, 2006) and as a minor compound of the extracellular secretion from living brown algae (Shibata, Hama, Miyasaki, Ito, \& Nakamura, 2006).

The compound $(Z, Z)-3,6$-nonadienal is one of the precursors of the cucumber-like aroma which is produced from fatty acid oxidation (Ma et al., 2007). The pleasant odour in cucumber has been attributed to $(E, Z)-2,6$-nonadienal, while two unsaturated aldehydes (2-hexenal and 2-nonenal) and three saturated aldehydes (ethanal, propanal and hexanal) were considered to contribute secondarily to overall flavour (Beaulieu and Baldwin, 2002; Beaulieu, 2005; Beaulieu \& Grimm, 2001; Palma-Harris, McFeeters, \& Fleming, 2002), but $(Z, Z)-3,6$-nonadienal was not recovered in Cantaloupe melons (Beaulieu \& Grimm, 2001). The NIL SC8-1 can be used as a model to avoid cucumber-like aroma in melons or to study the biosynthetic pathways of C6 and C9 unsaturated aldehydes and alcohols from linolenic precursors (Ma et al., 2007). Hydroxyperoxide lyases (HPL) cleave these C9 aldehydes (Tijet, Schneider, Muller, \& Brash, 2001). HPL form very unstable hemiacetals from hydroperoxides (HPO) generated by lipoxygenases from the polyunsaturated fatty acids, leading to the generation of aldehydes and aldehyde enols by spontaneous dissociation (Pech et al., 2008, chap. 13). The compound (E,Z)-2,6-nonadienal is a muskmelon-like or musky aroma and and (Z,Z)-3,6-nonadien-1-ol is a grassy boiled leaf-like aroma (Kourkoutas et al., 2006), Due to the reasons mentioned above, the former compounds can be easily obtained from $(Z, Z)-3,6$-nonadienal by isomerisation.

This aroma profile has multiple uses for the characterization of melon cultivars of the Inodorus Group, particularly of the 'Piel de sapo' type. For example, this profile can be used for present or future European protected geographical indications (PGI). Additionally, the compounds can be used to train and establish melon test panel and sensorial protocols, or to correlate sensory and instrumental parameters, for new or traditional melon cultivars (Pardo et al., 2000). Finally, the profile is useful for standardizing or developing new natural melon flavours and fragances; for the on-line control using the right sensors of fresh or processed melon products (Muñoz-Aguirre et al., 2007), such as fresh-cut halves, slices or cubes; or cremogenates, soups, canning balls, etc. This profile is also useful for developing new melon fruit spirits and distillates (Hernández-Gomez, Ubeda, \& Briones, 2003) with aroma volatiles closer to the fresh fruit aroma.

\section{Conclusions}

The aroma profile of this collection was composed of 24 compounds with predominance of aldehydes in non-climacteric melons, particularly hexanal and methanethiolate. The introgressions of PI 161375 studied also had higher levels of one ester and lower levels of octanal with no significant effects on the rest of the compounds of the profile. Three QTLs associated with ester and aldehyde biosynthesis were mapped, while another QTL was associated with the cucumber-like aroma $[(Z, Z)-3,6$ nonadienal].

\section{Acknowledgements}

This work was funded by Grants BIO-AGR06/02-0011 (Consejería de Educación y Cultura de la Región de Murcia), 05676/PI/ 07 and 00620/PI/04 (Fundación Séneca de la Región de Murcia), AGL2003-09175-C02-01 and AGL2003-09175-C02-02 from the Spanish Ministry of Education and Science and European Fund for Regional Development (FEDER, European Union). J. Obando was partly supported by a fellowship from the Spanish Ministry of Foreign Affairs (MAE-AECI). The authors are indebted to Ana Belén Pérez and Manuela Selma for sampling assistance and to Semillas Fitó S.A. (Barcelona, Spain) for providing the PS seeds.

\section{References}

Aubert, C., \& Pitrat, M. (2006). Volatile compounds in the skin and pulp of Queen Anne's pocket melon. Journal of Agricultural and Food Chemistry, 54, 8177-8182.

Baldwin, E. (2000). Flavor trivia and tomato aroma. Biochemistry and possible mechanisms for control of important aroma components. HortScience, 35, 1013-1022.

Baldwin, E. (2002). Fruit flavor, volatile metabolism and consumer perceptions. In M. Knee (Ed.), Fruit quality and its biological basis (pp. 89-106). Sheffield, UK: Sheffield Academic Press Ltd.

Baldwin, E., Goodner, K., Plotto, A., Pritchett, K., \& Einstein, M. (2004). Effects of volatiles and their concentration on perception of tomato descriptors. Journal of Food Science, 69, S310-S318.

Beaulieu, J. (2005). Within-season volatile and quality differences in stored freshcut cantaloupe cultivars. Journal of Agricultural and Food Chemistry, 53, 8679-8687. 
Beaulieu, J. (2006). Volatile changes in cantaloupe during growth, maturation, and in stored fresh-cuts prepared from fruit harvested at various maturities. Journal of the American Society of Horticultural Science, 131, 127-139.

Beaulieu, J. C., \& Baldwin, E. (2002). Flavor and aroma of fresh cut fruits and vegetables. In O. Lamikanra (Ed.), Fresh cut fruits and vegetables. Science, technology and market (pp. 391-425). Boca Ratón, Fla: ORC Press LLC.

Beaulieu, J. C., \& Grimm, C. C. (2001). Identification of volatile compounds in cantaloupe at various developmental stages using solid phase microextraction. Journal of Agricultural and Food Chemistry, 49, 1345-1352.

Beaulieu, J. C., \& Lancaster, V. A. (2007). Correlating volatile compounds, sensory attributes, and quality parameters in stored fresh-cut cantaloupe. Journal of Agricultural and Food Chemistry, 55, 9503-9513.

Beaulieu, J. C., \& Lea, J. M. (2006). Characterization and semiquantitative analysis of volatiles in seedless watermelon varieties using solid-phase microextraction. Journal of Agricultural and Food Chemistry, 54, 7789-7793.

Canoles, M. A., Beaudry, R. M., Li, C. Y., \& Howe, G. (2006). Deficiency of linolenic acid in Lefad7 mutant tomato changes the volatile profile and sensory perception of disrupted leaf and fruit tissue. Journal of the American Society for Horticultural Science, 131, 284-289.

Eduardo, I., Arús, P., \& Monforte, A. J. (2005). Development of a genomic library of near isogenic lines (NILs) in melon (Cucumis melo L.) from the exotic accession PI 161375. Theorethical and Applied Genetics, 6, 1-10.

Eduardo, I., Obando, J., Martínez, J. A., Alarcón, A. L., Arús, P., Álvarez, J. M., et al. (2007). Estimating the genetic architecture of fruit quality traits in melon (Cucumis melo L.) using a genomic library of near-isogenic lines. Journal of the American Society of Horticultural Science, 132, 80-89.

Fallik, E., Alkali-Tuvia, S., Horev, B., Copel, A., Rodov, V., Aharoni, Y., et al. (2001). Characterisation of 'Galia' melon aroma by GC and mass spectrometric sensor measurements after prolonged storage. Postharvest Biology and Technology, 22, 85-91.

Fernández-Trujillo, J. P., Obando, J., Martínez, J. A., Moreno, E., García-Mas, J., \& Monforte, A. J. (2008). Climacteric or non-climacteric behavior in melon fruit 2. Linking climacteric pattern and main postharvest disorders and decay in a set of near-isogenic lines. Postharvest Biology and Technology, 50, 125-134.

García-Esteban, M., Ansorena, D., Astiasarán, I., Martín, D., \& Ruiz, J. (2004). Comparison of simultaneous distillation extraction (SDE) and solid-phase microextraction (SPME) for the analysis of volatile compounds in dry-cured ham. Journal of Science and Food Agriculture, 84, 1364-1370.

Hattori, S. (2006). Study of new approach for evaluation of Japanese green tea using OASIS method. Bulletin of the Faculty of Agriculture Hokkaido University, 28(1), 85-120. eprints.lib.hokudai.ac.jp/dspace/bitstream/2115/8282/1/28(1)_2.pdf.

Hernández-Gomez, L. F., Ubeda, J., \& Briones, A. (2003). Melon fruit distillates: Comparison of different distillation methods. Food Chemistry, 82, 539-543.

Kondjoyan, N., \& Berdagué, J. L. (1996). A compilation of relative retention indices for the analysis of aromatic compounds. In Laboratoire de Flaveur et Diazol (Ed.), Clermont-Ferrand, France.

Kourkoutas, D., Elmore, J. S., \& Mottram, D. S. (2006). Comparison of volatile compositions and flavour properties of cantaloupe, Galia and honeydew muskmelons. Food Chemistry, 97, 95-102.

Lamikanra, O., \& Richard, O. A. (2002). Effect of storage on some volatile aroma compounds in fresh-cut Cantaloupe melon. Journal of Agricultural and Food Chemistry, 50, 4043-4047.
Ma, Y., Hu, X., Chen, J., Chen, F., Wu, J., Zhao, G., et al. (2007). The effect of freezing modes and frozen storage on aroma, enzyme and micro-organism in Hami Melon. Food Science and Technology International, 13, 259-267.

Moreno, E., Obando, J., Dos-Santos, N., Fernández-Trujillo, J. P., Monforte, A. J., \& García-Mas, J. (2008). Candidate genes and QTLs for fruit ripening and softening in melon. Theoretical and Applied Genetics, 116, 589-602.

Muñoz-Aguirre, S., Yoshino, A., Nakamoto, T., \& Morizumi, T. (2007). Odor approximation of fruit flavors using a QCM odor sensing system. Sensors and Actuators B-Chemical, 123, 1101-1106.

Muriel, E., Antequera, T., Petrón, M. J., Andrés, A. I., \& Ruiz, J. (2004). Volatile compounds in Iberian dry-cured loin. Meat Science, 68, 391-400.

Obando, J., Fernández-Trujillo, J. P., Martínez, J. A., Alarcón, A. L., Eduardo, I., Arús, P., et al. (2008). Identification of melon fruit quality quantitative trait loci using near-isogenic lines. Journal of the American Society of Horticultural Science, 133 139-151.

Obando-Ulloa, J. M., Moreno, E., García-Mas, J., Nicolai, B., Lammertyn, J., Monforte, J. A., et al. (2008). Climacteric or non-climacteric behavior in melon fruit 1 . Aroma volatiles. Postharvest Biology and Technology, 49, 27-37.

Obando-Ulloa, J. M. Nicolai, B., Lammertyn, J., Bueso, M. C., Monforte, A. J. \& Fernández-Trujillo, J. P. (2009). Aroma volatiles associated with the senescence of climacteric or non-climacteric melon fruit. Postharvest Biology and Technology, 52, 146-155.

Oms-Oliu, G., Odriozola-Serrano, I., Soliva-Fortuny, R., \& Martin-Belloso, O. (2008) The role of peroxidase on the antioxidant potential of fresh-cut 'Piel de Sapo' melon packaged under different modified atmospheres. Food Chemistry, 106 1085-1092.

Palma-Harris, C., McFeeters, R. F., \& Fleming, H. P. (2002). Fresh Cucumber flavor in refrigerated pickles: Comparison of sensory and instrumental analysis. Journa of Agricultural and Food Chemistry, 50, 4875-4877.

Pardo, J. E., Alvarruiz, A., Varón, R., \& Gómez, R. (2000). Quality evaluation of melon cultivars. Correlation among physical-chemical and sensory parameters. Journal of Food Quality, 23, 161-170.

Pech, J. C., Latché, A., \& Van der Rest, B. (2008). Genes involved in the biosynthesis of aroma volatiles and biotechnological applications. In B. Bruckner \& S. G. Wyllie (Eds.), Fruit and vegetable flavour: Recent advances and future prospects (pp. 254-271). Abington, Cambridge, UK: Woodhead Pub. Ltd.

Ruiz, J., Ventanas, J., Cava, R., Andrés, A. I., \& García, C. (1999). Volatile compounds of dry-cured Iberian ham as affected by the length of the curing process. Meat Science, 52, 9-27.

Shibata, T., Hama, Y., Miyasaki, T., Ito, M., \& Nakamura, T. (2006). Extracellular secretion of phenolic substances from living brown algae. Journal of Applied Phycology, 18, 787-794.

Tijet, N., Schneider, C., Muller, B. L., \& Brash, A. R. (2001). Biogenesis of volatile aldehydes from fatty acids hydroperoxides: Molecular cloning of a hydroperoxide lyase (CYP74C) with specificity for both the 9- and 13hydroperoxides of linoleic and linolenic acids. Archives of Biochemistry and Biophysics, 386, 281-289.

Villanueva, M. J., Tenorio, M. D., Esteban, M. A., \& Mendoza, M. C. (2004). Compositional changes during ripening of two cultivars of muskmelon fruits. Food Chemistry, 87, 179-185. 